\title{
Santiago Felipe Ramón y Cajal, ¿Padre de la Neurociencia o Pionero de la Ciencia Neural?
}

\author{
Santiago Felipe Ramón y Cajal, ¿Neuroscience Father or Pioneer of Neural Science? \\ *;** Jorge Eduardo Duque Parra; *John Barco Ríos \& ${ }^{* * * *}$ Félix John César Peláez Cortes
}

DUQUE, P. J. E.; BARCO, R. J. \& PELÁEZ, C. F. J. C. Santiago Felipe Ramón y Cajal, ¿Padre de la neurociencia o poinero de la ciencia neural? Int. J. Morphol., 29(4):1202-1206, 2011.

RESUMEN: El objetivo de este trabajo es demostrar, con base en referentes de la literatura neurocientífica, que Santiago Ramón y Cajal no fue padre de la Neurociencia, pero sí uno de los pioneros de la ciencia neural. Para corroborar dicha aseveración se consultó información variada sobre Ramón y Cajal en la que se indicara que él es uno de los pioneros de la Neurociencia y se contrastó con el tiempo en que se instauró la Neurociencia como disciplina; luego se comparó dicha información con aspectos variados de otros personajes que pudieron no ser llamados fundadores de la Neurociencia. Se concluye que Santiago Felipe Ramón y Cajal no es el padre de la Neurociencia, aunque sí se resalta el que es considerado uno de los pioneros de la ciencia neural.

PALABRAS CLAVE: Cajal; Historia; Neurociencia; Ciencia neural.

\section{INTRODUCCIÓN}

"El hombre cree con más disposición lo que prefiere que fuera cierto". Francis Bacon.

\section{La Neurociencia}

El término Neurociencia refleja la naturaleza interdisciplinaria de la moderna investigación del cerebro (Duque-Parra, 2001a), la cual incluye múltiples disciplinas aparentemente disímiles entre sí (Duque-Parra, 2001b), además de una amplia gama de técnicas seguramente mucho más diversas que cualquier otra rama de la ciencia (Blakemore, 1986) y con las que se puede contribuir a la comprensión del sistema nervioso (Duque-Parra, 2001b).

La Neurociencia se diferencia de otras ciencias cognitivas porque puede enunciar de manera más inequívoca sus interrogantes y verificar si se avanza o no en las soluciones, construyendo así los mejores puentes explicativos entre el nivel neuroquímico y el neuronal, pasando por el macroscópico, niveles que se hacen más complejos en la explicación de la percepción, la memoria y el aprendizaje, entre otras, además de sus alteraciones, ya que es el encéfalo humano el que se explica a sí mismo (Duque Parra, 2001b), y no sólo el encéfalo, pues la Neurociencia ha tenido contribuciones que han cimentado las bases para erigirse como un conjunto integrado de conocimientos sobre todo el sistema nervioso (Duque-Parra et al., 1999).

La Neurociencia es joven (Warwick Coen, 1986) y como término (Bear et al., 1998), pues se acuñó a finales de la década de 1960 (Bloom et al., 1999) y ya para 1970 se fundó la Society for Neuroscience, una asociación de neurocientíficos profesionales. La revolución de esta área de la ciencia se inició cuando científicos de disciplinas diferentes se dieron cuenta que la mejor esperanza de comprender la forma de funcionar del cerebro procedía de un enfoque interdisciplinario (Bear et al.); para ello, se dedicaron al desarrollo de un lenguaje común entre los neurobiólogos a través de una serie de conferencias sobre las ciencias del cerebro, como la del simposio "Mecanismos cerebrales y conciencia", promovido en 1952 por el Consejo Internacional de Organismos de Ciencias Médicas (UNESCO-OMS) (Rose, 1972).

Hasta la primera mitad del siglo XX no se hablaba de un área de la Neurociencia propiamente constituida, de-

\footnotetext{
* Programa de Medicina. Departamento de Ciencias Básicas. Grupo Neurociencia de Caldas. Universidad de Caldas, Manizales, Colombia.

*** Departamento de Ciencias Básicas Biológicas. Universidad Autónoma de Manizales, Manizales, Colombia.

**** Programa de Medicina. Departamento Básico Clínico. Universidad de Caldas, Manizales, Colombia.
} 
bido, al menos, a la distancia entre lo estructural y lo fisiológico. Con el desarrollo técnico, bioquímico, electrónico, imagenológico, entre otros, se favoreció el eslabonamiento morfológico y fisiológico y permitió, a partir de los años 1960, dar solidez a la Neurociencia (Duque-Parra, 2002).

Originalmente se puede reconocer a la Neurociencia como surgida en la década de 1950 (Duque-Parra et al.), aunque se cita que lo fue aproximadamente en los años 1960 (Blakemore). Por lo tanto, la Neurociencia solo tiene un corto aunque intenso período de trabajo de unos 50 años (DuqueParra, 2001b) aproximadamente, de ahí que sea una de las más rápidas áreas en crecimiento de la ciencia; tanto así que en 1971, 1100 científicos se congregaron en la primera reunión anual de su sociedad. Para 1997 participaron 27685 científicos en la reunión número 27 de la sociedad (Bloom et al.), lo que para ese tiempo resultó en un incremento de aproximadamente $2516 \%$ de sus participantes en 26 años y un $3415 \%$ de aumento para la trigésima quinta reunión realizada en el 2005, en la que ya se contaba con 37562 miembros (Society for Neuroscience, 2006).

En resumen, hacia finales de la década de 1960, con el término de Neurociencia se señaló el principio de una área en la cual cada una de las disciplinas neuromorfológicas, neurofisiológicas, neuroquímicas y neuropsicológicas, trabajaron sinérgicamente compartiendo un lenguaje común, unos conceptos comunes y una meta común con el fin de comprender mejor el cerebro (Bloom et $a l$.), además de otros componentes del sistema nervioso. Actualmente, la Neurociencia abarca la biología molecular de las células nerviosas, las bases biológicas de la conducta y la cognición normal y anormal (Bloom et al.), entre otras.

\section{Santiago Felipe Ramón y Cajal}

Cajal, uno de los más importantes científicos de habla hispana, contribuyó de manera sobresaliente al conocimiento de la anatomía microscópica del tejido nervioso, y se destacó por sus actitudes y valores, independencia de juicio, perseverancia en el estudio, búsqueda original y promoción de vocaciones (Ocampo Martínez, 2007).

Más conocido como Santiago Ramón y Cajal, nació el 1 de mayo de 1852 en Petilla de Aragón -España- y murió en 1934. Su obra La textura del sistema nervioso del hombre y los vertebrados (Ramón y Cajal, 1899) es, según el premio Nobel de Medicina y Fisiología de 1981, David H. Hubel, el más importante trabajo publicado en todos los tiempos sobre Neurobiología científica (Velayos-Jorge et al., 2003). La obra de Cajal ha resistido airosa el paso del tiempo, prueba de ello es que Cajal es todavía el científico más citado en la bibliografía mundial (Society for Neuroscience;
Velayos-Jorge et al.), por encima de Albert Einstein (Delgado-Bona, 2003) o el único científico comparable a este último (Montes-Santiago, 2006).

La genialidad de Cajal se alimentó de su férrea voluntad, que para fortuna de los neurólogos, neurobiólogos, neuroquímicos y neurocientíficos en general, se centró apasionada y obsesivamente en el estudio del sistema nervioso (Delgado-Bona) fundando la doctrina neuronal (Fernández \& Breathnach, 2001). A Cajal se le reconoce su grandiosa y extensa aportación a la ciencia neural, la cual con el paso del tiempo entró a ser parte de la Neurociencia, tanto así que hoy en día, 100 años después de sus aportes originales, sigue siendo citado prolíficamente en múltiples artículos de Neurociencia (Díaz Güell, 2003).

Sobre Ramón y Cajal y su relación con la Neurociencia se ha indicado que, por identificar la neurona como unidad básica del sistema nervioso, fue el padre cimentador (DeFelipe, 2004), el verdadero iniciador y el fundador de la Neurociencia moderna (Swanson et al., 1999; Delgado García, 2000; DeFelipe, 2002), uno de los más reconocidos padres de las neurociencias actuales (Mora, 2004). Incluso, en revistas comerciales se ha enfatizado el que merezca ser reconocido por la comunidad científica internacional como el padre de la Neurociencia moderna (Díaz Güell). También se ha indicado que como insigne premio Nobel de Medicina y Fisiología (1906) y primer laureado Nobel de España (DeFelipe, 2004), contribuyó de manera importante a las Neurociencias (Freire Mallo, 1998-1999) y que numerosos investigadores siguieron su ejemplo comprobando y ampliando sus teorías en prácticamente todos los campos de la Neurociencia (DeFelipe, 2004).

\section{Cajal y la ciencia neural}

El término neurona se asignó a la célula nerviosa y fue acuñado por Wilhelm von Waldeyer-Hartz, profesor de anatomía y patología en Berlín, en 1891 (Illing, 2002; Rubia, 1992; Ramón y Cajal \& Tello y Muñoz, 1955; Mazzarello, 2002); sin embargo, los primeros pasos para la fijación de pequeñas muestras de tejido nervioso las logró el anatomista alemán Otto Deiters, quien pudo ver las prolongaciones filamentosas que salían del cuerpo neuronal, hoy conocidas como dendritas y axón (Illing). Aunque la publicación de Schäefer en 1878 contribuyó a lo que se conocería más adelante como doctrina neuronal, la aparición de esta idea se asocia a menudo a las observaciones tempranas de His (el año 1886) y Forel (el año 1887) y a los esfuerzos sostenidos de Ramón y Cajal (Mazzarello). Con ello, se ha completado poco más de un siglo de la moderna ciencia neural, desde que Cajal proveyó una crítica evidencia de esta doctrina (Albright et al., 2000). 
El término "sinapsis" fue acuñado en 1897 por el laureado Premio Nobel de Medicina y Fisiología de 1932, Sir Charles Scott Sherrington (Illing; Palacios, 1984; Somjen, 1986; López Piñero; 2002), al resumir las ideas de Cajal acerca de la conexión entre las neuronas por no continuidad (Somjen, 1986) y para quien la sinapsis explicaría el retraso en la conducción de los impulsos nerviosos (Palacios; Somjen). Tales asignaciones y hallazgos fortalecieron la concepción neuronal de Ramón y Cajal, que fueron corroboradas en 1955 con el advenimiento de la microscopía electrónica, cuando De Robertis, Bennett y Palade ampliaron el conocimiento sobre los "contactos" neuronales al esclarecer sus elementos básicos constitutivos: las vesículas sinápticas y sus neurotransmisores (Albright et al.; Somjen; Ralston, 1998; Toledano Gasca; 1985), lo que permitió refutar la teoría que desde décadas atrás consideraba el sistema nervioso como un sincitio (Somjen): la red difusa de Golgi (Mazzarello). Téngase en cuenta que Cajal no vio nunca una sinapsis (Toro Gonzáles, 2000), aunque sí las infirió. Sus trabajos sirvieron de "caldo de cultivo" para los neurocientíficos, pues numerosos investigadores siguieron el ejemplo establecido por Ramón y Cajal, comprobando y ampliando sus teorías en prácticamente todos los campos de la neurociencia (Swanson et al.).

Para realizar este estudio se consultó información variada, referida en el aparte de referencias bibliográficas, sobre Ramón y Cajal en la que se indicara que él es uno de los padres o pioneros de la Neurociencia y de la ciencia neural; luego se contrastó dicha información con el tiempo en que se instauró la Neurociencia con nombre propio, y se comparó con aspectos variados de otros personajes que pudieron o no ser llamados fundadores de la Neurociencia.

\section{DISCUSIÓN}

Recientemente un autor (Toro Gonzáles) supuso erradamente que por haberse hecho mención de las primeras trepanaciones, o por las consideraciones filosóficas hechas sobre el cerebro y la mente, o por los inicios de la Medicina, o por los trabajos histológicos de Cajal, o por la Neurología de Charcot, o por la Neurocirugía de Harvey o por la Neuropatología, se sentaron las bases de la Neurociencia o que eso es Neurociencia; incluso se ha hecho una subdivisión artificial en la que se enmarca la Neurociencia en el mundo entre los años 1850 y el 2000 (Pevsner, 2005).

El estudio del cerebro -componente del sistema nervioso- es tan antiguo como la propia ciencia (Bear et al.); sin embargo, hasta la primera mitad del siglo pasado, la neuroanatomía y la neurofisiología fueron las ramas de las ciencias básicas que más aportaron al conocimiento funda- mental del sistema nervioso (Duque-Parra, 2002), en las que Cajal aportó fundamentalmente en lo concerniente a la neurohistología, descubrimientos que han sido catalogados como trabajos neurocientíficos notables (Mora, 2002). Sin embargo, los aportes de este científico se dieron antes de existir la Neurociencia como tal, pues Cajal murió en 1934.

Aunque en la construcción de la Neurociencia han participado múltiples personalidades, no es del todo cierta la expresión de que Cajal fue uno de los más brillantes neurocientíficos (Bosch, 2001) y que su contemporáneo Charles Sherrington fue uno de los grandes científicos de la historia de la neurociencia (Barco, 2010). La aseveración que se hace sobre Cajal puede considerarse un error, aunque no se puede negar la grandiosa y extensa aportación a la ciencia neural que realizó Ramón y Cajal, tanto que en múltiples artículos recientes en el campo de la Neurociencia es citado frecuentemente, casi 100 años después de sus aportes originales. Es un error conceptual, aunque de buena intención, nominar a Cajal como pionero y/o padre de la Neurociencia, para algunos de manera indiscutible (Barco), puesto que vivió entre 1852 a 1934 , mientras que la Neurociencia surgió en la década de 1950. Charles Scott Sherrington vivió entre 1857 a 1952 y ha sido considerado como pionero de la Neurociencia (Mora, 2002), lo que concuerda con los datos que se tienen sobre la época en que surgió esta nueva disciplina. Por lo tanto, en concordancia con los datos suministrados, sería más adecuado considerar a Sherrington como pionero de la Neurociencia y no a Cajal.

Hubo también otros científicos contemporáneos de Cajal que lograron grandes descubrimientos y realizaron importantes y trascendentales aportes a las distintas disciplinas de la ciencia neural, sin embargo no han merecido el calificativo de pioneros de la Neurociencia. Tal es el caso del científico Johann LW Thudichum -1829-1901-, considerado el fundador de la Neuroquímica por haber descrito, en 1884, los constituyentes químicos del cerebro, incluyendo los cerebrósidos (Eling, 2001).

Por consecuencia con lo planteado atrás, Santiago Ramón y Cajal, que vivió hasta 1934, no pudo haber sido el padre de la Neurociencia, que surgió en la década de 1950, pero sí es uno de los pioneros de la ciencia neural, pues proporcionó una evidencia crítica para la doctrina neuronal, como es la idea de que las neuronas sirven como unidades de señales funcionales del sistema nervioso y que las neuronas se conectan una a otra en forma precisa. En las primeras décadas del siglo XX, cuando aún vivía Cajal, la Neuroanatomía y la Neurofisiología eran las ramas más desarrolladas y las que más aportaban al conocimiento de la estructura y funcionamiento del sistema nervioso, pero todavía no se podía hablar de Neurociencia, puesto que aún 
existía una distancia muy amplia entre los hallazgos fisiológicos y su base estructural.

Si se considera el término Neurociencia de manera holística y atemporal, entonces debería considerarse como Neurociencia a cualquier hallazgo o contribución científica o filosófica que se halla dado sobre consideraciones del sistema nervioso en cualquier época de la humanidad, como los descubrimientos neuroanatómicos que hizo Leonardo da Vinci -1452-1519- hace unos 500 años, alcanzando un conocimiento científico más preciso del cerebro (Mora, 2002), pero no por ello fue neurocientífico, aunque se considera que sus trabajos contribuyeron a la Neurociencia (Pevsner, 2002). También valdría entonces considerar a los Egipcios del siglo $\mathrm{V}$ aC, a los griegos helenísticos, a los romanos y a ciertos personajes del Medioevo, todos los cuales contribuyeron a la comprensión de diversas partes del sistema nervioso, en especial del cerebro. Sin embargo, para ese entonces no existía el término Neurociencia y tampoco había una integración de las áreas de la ciencia para construir un arsenal teórico y técnico para una comprensión más objetiva de los eventos que acontecen con la actividad del sistema ner- vioso. A los múltiples contribuyentes de épocas anteriores a 1950 los reconocemos desde sus respectivas áreas asociadas a la Neurociencia, integradas al conocimiento del dónde (neuroanatomistas) y el cómo (neurofisiólogos) de los procesos conductuales.

Parafraseando al neurocientífico Samir Zeki, el hecho de que hoy seamos capaces de formular mucho mejor los problemas y de encontrarles solución, no altera el hecho de que el objeto de la investigación de la Neurociencia en el futuro y de las ramas que la generaron en el pasado, tengan una misma raíz.

\section{CONCLUSIÓN}

Santiago Felipe Ramón y Cajal no es ni padre, ni fue pionero de la Neurociencia; sin embargo, por sus enormes contribuciones neuro-histológicas, sí es considerado como uno de los pioneros de la ciencia neural, elemento componente de la Neurociencia.

DUQUE, P. J. E.; BARCO, R. J. \& PELÁEZ, C. F. J. C. Santiago Felipe Ramón y Cajal, ¿Neuroscience father or pioneer of neural science? Int. J. Morphol., 29(4):1202-1206, 2011.

SUMMARY: The present work on Santiago Ramón y Cajal aims to demonstrate on the basis of neuroscientist literature, that Santiago Ramón y Cajal was not the father or founder of Neuroscience, but is considered a neural science pioneer. In order to corroborate this statement, varied information on Ramón y Cajal was consulted within Neuroscience to determine if Ramón Y Cajal is one of the Neuroscience pioneers, and contrast that information with the beginnings of neuroscience as a discipline. Subsequently this information was compared with various aspects of other important figures who were not named Neuroscience founders. In conclusion Santiago Felipe Ramón and Cajal if not the father of Neuroscience, though it should be noted he is considered one of the pioneers of neural science.

KEY WORDS: Cajal; History; Neuroscience; Neural science.

\section{REFERENCIAS BIBLIOGRÁFICAS}

Albright, T. D.; Jessell, T. M.; Kandel, E. R. \& Posner, M. I. Neural Science: A century of progress and the mysteries that remain. Cell, 100:S1-S55, 2000.

Barco, A. La materia de los recuerdos: circuitos neuronales y cascadas moleculares. Mente y Cerebro, 40:24-33, 2010.

Bear, M. F.; Connors, B. W. \& Paradiso, M. A. Neurociencia. Explorando el cerebro. Barcelona, Masson-Williams \& Wilkins, 1998.

Blakemore, C. La naturaleza de la explicación en el estudio del cerebro. En: Warwick Coen, C. (Ed.). Las funciones del cerebro. Barcelona, Editorial Ariel S.A., 1986. p.242-66.

Bloom, F. E.; Fischer, B. A.; Landis, S. C.; Roberts, J. L.; Squire, L. R. \& Zigmond, M. J. Fundamentals of Neuroscience. En: Zigmond, M. J.; Bloom, F. E.; Landis, S. C.; Roberts, J. L. \& Squire, L. R. (Eds.). Fundamental Neuroscience. San Diego, Academic Press, 1999. pp.3-8.
Bosch, X. The brain in Spain. Nature, 409(6819):451, 2001.

DeFelipe, J. Cerebro y cultura. En: Mora, F. (Ed). Esplendores y miserias del cerebro. Madrid, Fundación Santander Hispano, 2004. pp.61-100.

DeFelipe, J. El cerebro en el espacio. Mente y cerebro, 1:72-81, 2002.

Delgado-Bona, G. Conferencia: Homenaje de la SENP a Santiago Ramón y Cajal. Rev. Neurol., 37(1):89-91, 2003.

Delgado García, J. M. Neurociencia para pobres. Claves de Razón Práctica, 102:42-7, 2000.

Díaz Güell, L. Ramón y Cajal, padre de la Neurociencia moderna. Ronda Iberia, 4:54, 2003.

Duque-Parra, J. E. Functional neuroanatomy: the first daugther of 
neuroscience and the mother of neural science. Anat. Rec., 265(6):250-3, 2001a.

Duque-Parra, J. E. Las Neurociencias: orígenes y conceptos. Rev. Med. Cal., 15:29-35, 2001b.

Duque-Parra, J. E. Elementos neuroanatómicos y neurológicos asociados con el cerebro a través del tiempo. Rev. Neurol., 34(3):282-6, 2002.

Duque-Parra, J. E.; Morales Parra, G. \& Díaz Zapata, J. J. Aspectos históricos, evolutivos y conceptuales de las neurociencias. Rev. Med. Risaralda, 1:15-20, 1999.

Eling, P. Neuro-anniversary 2001. J. Hist. Neurosci., 10(1):2-5, 2001.

Fernández, N. \& Breathnach, C. S. Luis Simarro Lacabra (18511921): from Golgi to Cajal through Simarro, via Ranvier? J. Hist. Neurosci., 10(1):19-26, 2001.

Freire Mallo M. Legado Cajal. Memoria científica. CSIC Instituto Cajal. Madrid, Consejo superior de investigaciones científicas, 1998-1999. pp.263-89.

Illing, R. B. De la trepanación a la teoría de la neurona. Mente y Cerebro, 1:82-9, 2002.

López Piñero, J. M. Cajal y la estructura histológica del sistema nervioso. Investigación y Ciencia Temas, 29:72-9, 2002.

Mazzarello, P. Camilo Golgi y la reacción negra. Investigación y Ciencia Temas, 29:64-71, 2002.

Montes-Santiago, J. El encuentro de Einstein y Cajal (Madrid, 1923): un olvidado momento estelar de la humanidad. Rev. Neurol., 43(2):113-7, 2006.

Mora, F. El reloj de la sabiduría. Tiempos y espacios en el cerebro humano. Madrid, Alianza Editorial, 2002.

Mora, F. El reloj de la sabiduría. Tiempos y espacios en el cerebro humano. Madrid, Alianza Editorial S. A., 2004.

Ocampo Martínez, J. El legado ético y epistemológico de Santiago Ramón y Cajal. XVI Congreso Panamericano de Anatomía, San José, Costa Rica, 2007.

Palacios, P. E. Breves comentarios sobre Neurobiología celular y del desarrollo. Caracas, Instituto Internacional de Estudios Avanzados, 1984.

Pevsner, J. Leonardo da Vinci`s contributions to Neuroscience. Trends Neurosci., 25(4):217-20, 2002.

Pevsner, J. Leonardo da Vinci, neurocientífco. Mente y Cerebro, 13:78-82, 2005.
Ralston, H. J. 3rd. Untying the gordian knot: contemporary studies of neuronal organization. Anat. Rec., 253(5):139-42, 1998.

Ramón y Cajal, S. Textura del sistema nervioso del hombre y de los vertebrados. Madrid, Imprenta y Librería de Nicolás Moya, 1899.

Ramón y Cajal, S \& Tello y Muñoz, J. F. Elementos de Histología normal y de técnica micrográfica. México D.F., Editora Nacional, 1955.

Rose, S. El futuro de las ciencias del cerebro. En: Nathan, P.; Luria, A. R.; Herbert, M.; Kempt, M.; Horn, G. \& Bateson, P. (Eds). El fantástico mundo del cerebro. Caracas, Monte Avila editores, 1972. pp.127-38.

Rubia, F. J. La neurona y las células de glía. En: Tresguerres, J. F. A.; Aguilar Benítez de Lugo, E.; Gallego Fernández, A.; GilLoyzaga, P.; Lahera Julia, V. \& Martínez Verano, J. A. (Eds). Fisiología Humana. Madrid, McGraw-Hill Interamericana, 1992. pp.17-22.

Society for Neuroscience. Navigating a changing landscape. Annual Report 2006. Disponible en: http://www.sfn.org/skins/ main/pdf/AnnualProgressReport/2006ar.pdf

Somjen, G. G. Neurofisiología. Buenos Aires, Médica Panamericana, 1986.

Swanson, L. W.; Lufkin, T. \& Colman, D. R. Organization of Nervous systems. En: Zigmond, M. J.; Bloom, F. E.; Landis, S. C.; Roberts, J. L. \& Squire, L. R. (Eds). Fundamental Neuroscience. San Diego, Academic Press, 1999. pp.9-37.

Toledano Gasca, A. La conexión morfofuncional de las neuronas: sinapsis y neurotransmisión. Bogotá, Merck, 1985.

Toro Gonzáles, G. Origen y evolución de la Neurociencia. Rev. Acad. Colomb. Cienc., 24(92):401-15, 2000.

Velayos-Jorge, J. L.; Hernández-Roca, J. J.; Moleres-Echeverría, F. J. Neurobiología del sueño: Ramón y Cajal y la neurociencia actual. Rev. Neurol., 37(5):494-8, 2003.

Warwick Coen, C. Introducción. En: Warwick Coen, C. (Ed.). Las funciones del cerebro. Barcelona, Editorial Ariel S. A., 1986. p.11-9.

Dirección para correspondencia:

Jorge Eduardo Duque Parra

Programa de Medicina. Departamento de Ciencias Básicas Universidad de Caldas. Manizales

COLOMBIA

Email: jorge.duque_p@ucaldas.edu.co

Recibido : 02-06-2011

Aceptado: 22-09-2011 